\title{
FUTBOLANO, \\ LA MOLECULA QUE ASOMBRA AL MUNDO
}

Raúl Paredes Medina

\section{INTRODUCCION}

Los químicos Rick Smalley, de la Universidad de Rice, en Houston, y Harry Kroto de la Universidad de Sussex, en Inglaterra, dieron a conocer la existencia de una nueva molécula de carbono: un monstruo compuesto por 60 átomos con la particularidad de noestar configurada en una cadena. Según los investigadores, era todo un balón de fútbol que podía haber sido diseñado por el mismísimo Buckminster Fuller, el creador de la cúpula geodésica que se encuentra en el Epcot Center de Florida (EE. UU.)

Actualmente, estos científicos siguen defendiendo con saña su balón molecular. Pero nadie lo ha visto, sólo se sabe que existe. Dicen que está en todas partes: se desliza por el aire, arde en los hornos, titila en lallama de las velas encendidas. Fuera de la tierra existe aún en mayor cantidad. Smalley comenta: "Puede ser una de las moléculas más abundantes y antiguas en el cosmos; su edad podría remontarse a la primera generación de las estrellas, hace unos 10000 millones de años".

Una de las primeras cosas que hicieron Smalley y Kroto fue darle un nombre a su molécula. Se consideraron los nombres de: esfereno, boleno, carbofútbol... Todos eran demasiado prosaicos. Al final decidieron honrar a quien lo merece, yacordaron ponerle el nombre de buckminsterfullereno, bucky para abreviar. Sin embargo, hayquien hapreferido dejarla en futbolano, en plan familiar, o fullereno en plan formal.

\section{DESCUBRIMIENTO}

En 1984, Smalley investigaba en Rice, las propiedades de enlace de variosátomos. Su método consistía en secar un elemento, silicio por ejemplo, con un láser y luego, con ayuda del espectrómetro, observar cómo volvían a unirse lentamente los átomos. Mientras, en la localidad inglesa de Sussex, Kroto estudiaba el enlace atómico del carbono. Fusionando químicamente sus muestras, había conseguido crear una cadena de carbo- nos de siete átomos. Más tarde, en los espectros de polvo interestelar, encontró el indicio de una molécula que contenía nueve átomos de carbono. Estoola convertía en una de las moléculas más grandes, jamás halladas en el espacio.

Durante una visita a Rice, en la primavera de 1984, Kroto quedó impresionado con el método de Smalley. Le sugirió que intentara secar un carbono pequeño, pues con un experimento de este tipo podrían simularse las condiciones de la atmósfera exterior de estrellas ricas en carbono; esa especie de horno en el cual creía que se formaban las cadenas de carbono celestes. Smalley tomó en consideración la sugerencia, y dijo a Kroto que se pondría en contacto con él si conseguía algo. A fines de 1985, Kroto recibió la llamada. "Estaba tan emocionado, que me presenté en Houston en dos dias".

Smalley mediante un aparato impresionante de vaporización por láser había logrado pulverizar pequeñas fracciones de carbono en el interior de una cámara de vacío, otro láser hacía saltar los electrones de algunos agregados dejándolos con carga positiva. Luego, un campo eléctrico atrapaba los agregados cargados y los aceleraba en el espectrómetro de masa. Dado que los agregados más pesados son más lentos, tardan más tiempo que los ligeros en llegar al espectrómetro. Este aparato registraba cuántos agregados van llegando, y cuántos se han formado en cada tamaño.

Llegados a este punto, el asunto cobraba interés. Si las moléculas se movieran por azar, al espectrómetrollegarian agregados de carbono de todos los tamaños. Pero Krotoy Smalley vieron que el carbono se agrupaba casi exclusivamente en haces atómicos de igual número. Y, aún más extraño, el agrupamiento que parecían preferir era el agregado de 60 átomos. Estos resultados desafiabanla leyde probabilidades. Lapregunta era: por qué precisamente 60 ?

La noche antes de que Kroto regresara a Ingla- 
terra, resolvieron el misterio. Especulando sobre la forma que tendría este $\mathrm{C}_{60}$ si pudieran verlo de cerca, Kroto sugirió que podría ser la de una esfera. "El $\mathrm{C}_{60}$ no era reactivo", explica Smalley. "Una vez que llegaba a los 60 átomos, se mantenía ahí. Esto nos sugirió la idea de la esfera, pues la mayoría de las formas moleculares tienen extremos colgantes que atraerían a otros átomos o moléculas".

Pero, cómo se forma una esfera de carbono? El grafito, que es la forma común más conocida del carbono, estáhecho de moléculas hexagonales unidas en láminas. Kroto recuerda que, años atrás, hizo una esfera de cartulina para sus hijos, y su estructura, como la de las cúpulas de Buckminster Fuller, consistía en varios pentágonos y hexágonos colocados en una semiesfera. Aunque el grafito carece de los pentágonos necesarios, el paralelismo es notable. "Además", señala Smalley, "los átomos de carbono no se limitana la forma hexagonal: cuando la temperatura es lo bastante alta, ensayan muchas y muy diversas ordenaciones, buscando aquellas que les otorgue una mayor estabilidad".

Esa noche, al volver a casa, Smalley empezó a construir bolas geodésicas con papel. Recortó un pentágono y le pegó 5 hexágonos alrededor. "Tenía forma de cuenco. Después construyó otro anillo y se emocionó, porque aquello era una semiesfera. Contó los vértices y vio que tenía 30: con 60 tendría una esfera completa. Cortó y pegó más figuras, yal fin, tuvo un pentágono y un hueco pentagonal en la parte superior. Eureka!". En posteriores sesiones de recortadoy pegado, se demostró que se podian haceresferas geodésica, usando desde 20 átomos hasta cientos de ellos. Una característica de estas esferas es que la estructura siempre tiene 12 pentágonos. Si hay alguno más, la bola no se cierra, ya medida que aumenta el número de hexágonos, la esfera tiende a aplanarse alrededor de los pentágonos.

Pero el $\mathrm{C}_{60}$, con sus 20 hexágonos, es perfectamente esférico. Es también el más pequeño de los futbolanos, en el cual ninguno de los pentágonos se tocan.

Smalley y Kroto anunciaron su descubrimiento mediante un artículo en la revista Nature. Entonces fue cuando le dieron el nombre de futbolanos, no sólo a la molécula, sino a toda la familia de agregados esférico. Sin embargo, para algunos de sus colegas, la novedad no era digna ni de aparecer en el tablón de anuncios.

\section{REFUTACION}

La mayor refutación llegó de los laboratorios Exxon, en Annandale, Nueva Jersey. Alli un equipo de químicos estaba utilizando métodos similares de evaporación, y también produjeron agregados de $\mathrm{C}_{60}$; pero no en las cantidades de Smalley y Kroto. Donald Cox, de Exxon, comenta: "En los indicios de nuestros espectros de masa, posiblemente habria el doble de agregados de $\mathrm{C}_{60}$ que de otro tipo, pero no diez veces más". El equipo de Exxon creía tener una explicación más plausible que la desusada agrupación de átomos. Estaban seguros de que las unidades de $\mathrm{C}_{2}$ formaban cadenas, llamadas polienos, de estructura similar a la de los polímeros, que parecen cordones enrollados. Los polienos son moléculas no reactivas, lo cual satisface el requisito de la estabilidad. Y, lo que es más importante, mantenían que el enlace cruzado de los cordones de pares de carbonos explicaba por qué los átomos evaporados con láser parecian preferir siempre una agrupación de igual número.

Los investigadores de Exxon no descartaban la idea del futbolano, pero se oponían al triunfalismo con que SmalleyyKrotola presentaban en Nature. Ellos mismos, en un artículo posterior, sostuvieron que las teorías acerca del futbolano eran, cuando menos, incompletas. Tambiénespeculabanconlaposibilidaddequelaformación de los agregados de $\mathrm{C}_{60}$ de Smalley y Kroto no se debiera a una propiedad del carbono, sino del láser. Proponían que un cambio en la frecuencia de la carga de fotones implicaría otro cambio en el tamaño de los agregados resultante. Smalley y Kroto no aceptaron de buena gana esa contestación. "Creemos que no reflexionaron bien sobre lo que proponiamos", opina Smalley. Pero sus críticos tenian un punto a favor, las únicas pruebas de Kroto y Smalley eran los garabatos del espectrómetro y una bola hecha con recortes de papel.

\section{DEFENSA}

Lo que necesitaban los descubridores era una foto con la cual acallar a sus detractores. Para ello contaban con el microscopio de perforación y barrido, que utiliza una aguja cuya punta tiene poquísimos átomos, de modo que pueda trazar el contorno de objetos minúsculos; un ingeniero que hahecho realidad la fotografía atómica. Sin embargo, Bucky resultó enormemente tímida. Los investigadores de los laboratorios Bell AT \& Tya intentaron fotografiar, sin resultado, estas esferas. Colocaron los agregados de carbono evaporados con láser, incluido el $\mathrm{C}_{60}$, sobre una superficie de oro. Pero para que el microscopio pueda hacer un dibujo preciso,, la muestra y la superficie deben interactuar atómicamente, y originar un estado electrónico que guie la aguja. Por desgracia, los agregados de carbono tienen muy pocos extremos libres, cuando los tienen, con los que el oro pueda unirse; asi que ambos materiales no entran en contacto. "La única fotografía que tenemos hasta ahora", comenta Smalley, "es la de un terreno rocoso de desechos de carbono'. 
Al no tener el "corpus delicti", Smalley y Kroto empezaron abuscar pruebas circunstanciales. Sometieron los compuestos de heliocarbono a potentes emisiones de láser; después estudiaron cómo se separa la molécula, mediante las lecturas del espectrómetro de masa. Descubrieron que descomponer el $\mathrm{C}_{60}$ era difícil, ya que las pequeñas moléculas se separaban una por una.

Smalley explica el proceso: "El $C_{60}$ se convertía en $\mathrm{C}_{58}$, y quedaba una pequeña molécula de $\mathrm{C}_{2}$ flotando en el espacio. Si se convertía en $\mathrm{C}_{56}$, yasí sucesivamente". Sin embargo, esto sólo hacía disminuir la molécula hasta $\mathrm{C}_{32}$; en ese punto, quedaba inevitablemente destruida. Smalley y Kroto llegaron a la conclusión de que el láser conseguía evaporar las moléculas de $\mathrm{C}_{2}$ de la superficie del futbolano, el cual volvería a cerrarse, consiguiendo la estructura correspondiente más pequeña. Pero al llegar a los 32 átomos, el futbolano estaría bajo tal tensión que perder otro $\mathrm{C}_{2}$ lo haría reventar.

Su siguiente labor fue la de demostrar que los futbolanos eran esféricos. Para ello, hirvieron el grafito puro en una sal metálica, antes de ponerlo ante el láser. Así, cuando evaporaban el grafito, estaban evaporando tanto átomos de metal como de carbono. Por ello, los agregados que se formaran tendrían un ion del metal, que tal vez se quedara atrapado-dentro de la estructura. Para comprobar que la trama de carbono estaba, efectivamente, envolviendo al ion, los investigadores volvieron a utilizar el láser. Si el ion estaba realmente donde ellos creían, la expulsión de $\mathrm{C}_{2}$ se desarrollaría de la manera anterior, con la diferencia de qu la molécula se destruiría antes de llegar al $\mathrm{C}_{32}$. Esto ocurriria porque, en un determinado punto, la estructura se encogería lo bastante como para presionar al ion en su interior.

$\mathrm{Si}$ efectivamente existieran los futbolanos, Smalley y Kroto creen que sería una de las moléculas más comunes, formadas junto con el más corriente de los materiales carboníferos: el hollín. Para la mayoría de la gente el hollin no tiene mucho interés, pero los tecnólogos de la combustión no piensan lo mismo. Estos ingenieros invierten buena parte de sus energías en intentar conocer a fondo la producción de hollín, para eliminarla o para incrementarla. Por ejemplo, en un horno se necesita hollín para transferir calor desde la llama al agua o al aire que están siendo calentados. En cambio, no puede haber hollín en el motor de un avión. Si se permite la irradiación de calor a través del motor, el hollín puede deteriorar o destruir elementos muy delicados.

Es curioso que, sin embargo, nadie conozca exactamente la formación del hollín. Es bastante fácil recoger el material en el aire una vez que ha sido producido por la llama, y hacer un análisis de sus características químicas. Pero esto sólo descubre al hollín como producto final, no durante su proceso de formación, átomo porátomo. Kroto y Smalley creen qu la existencia del $\mathrm{C}_{60}$ podría servir para explicar cómo se forman las moléculas de hollín. Su teoría es que el futbolano al principio sólo es un conjunto de moléculas de carbono, unidas en láminas bidimensionales. Estas láminas tienen terminaciones de enlace molecular en sus extremos, yes allí donde los carbonos van uniéndose enformas pentagonales yhexagonales. Estas dos formas moleculares hacen que la lámina se curve, y cuantas moléculas se añadan, más pronunciada será la curva. Esto sucede hasta que la parte superior se cierra y ya tenemos futbolano.

Sin embargo, el proceso no es tan sencillo. La forma del futbolano puede verse influida por muchos factores: temperatura, composición del gas o química del combustible. Según Smalley y Kroto, la probabilidad de que se cierren las moléculas de carbono que van apareciendo y forman un futbolano acabado, es de una entre 10000. En las demás, los extremos en crecimiento pasarán por encima de los extremos opuestos, haciendo un rizo. Entonces la molécula empezará a crecer sin control, añadiendo más y más capas en espiral, como una bola de nieve bajando por una ladera. La molécula gigante que resulta de este proceso, formada porcientos o miles de átomos, es la que conocemos como hollín. Y, perdidos entre este desecho de carbón, estarian los futbolanos perfectos.

Estos dos investigadores creen que una de las productoras más corrientes de futbolano es la llama de una vela. En ella se puede observar, junto a la mecha, un pequeño resplandorazul, que es la zona de oxidación de la llama. El aire que hay alrededor, mezclado con la emanación del sebo caliente, se quema con el calor. La temperatura de esta zona azul puede sobrepasar los 3 400 grados. Smalley comenta: "Aquí, a esta altísima temperatura, donde puede pensarse que cualquier molécula orgánica se rompería en pedazos, es precisamente donde se da un proceso increíblemente rápido de formación de moléculas gigantes, con cientos de miles de átomos de carbono. Se hacen rápidamente incandescentes, y forma, el color amarillo de la llama".

Pero según Lawrence Ebert, otro químico de Exxon, y Michael Frenklach del Estado de Pennsylvania, Kroto y Smalley han errado en su interpretación del tamaño y forma del hollin. Ebert sostiene que hay infinidad de artículos publicados que ofrecen explicaciones plausibles de que el hollín está formado convencionalmente; es decir, en láminas bidimensionales. Y añade que Smalley y Kroto deberían tomarse la molestia de leerlos. En un rasgo sorprendente de ingenuidad, Smalley admite que parte de esa literatura se le ha escapado. 


\section{POLEMICA}

En 1989 la polémica se avivó durante una conferencia sobre el carbono celebrada en Pennsylvania. Kroto presentó una ponencia en la que exponía sus teorías y las de Smalley acerca de Bucky. Esta ponencia tuvo su contestación en otra, presentada porEbert: "Sólo porque algo sea sencillo y atrevido, no tiene que ser necesariamente correcto". Aunque la ponencia de Ebert mantenía un tono científico imparcial, a Kroto le disgustó este reto público. Sin embargo, a Smalley no le causaron problemas los golpes profesionales de Ebert. "En lo que a mí respecta, no valía la pena entrar en una gran discusión científica con esa gente, Nosotros presentamos nuestras teorías de un modo muy humilde, de tal manera que ellos pueden aceptarlas o no".

La opción de no aceptarlas parecía ser mayoritaria. Posiblemente, el más fuerte de los argumentos en contra del futbolano sea que el crecimiento de las espirales es demasiado lento como para explicar la aparición del hollín. Las láminas de carbono tienen muchos puntos en sus extremos donde pueden fijarse nuevos átomos, y cuando más grande se haga la lámina, más puntos se añaden. Pero las espirales son formas relativamente cerradas, y sólo la punta de formación de éstas ofrece lugares de enlace. La espiral necesitaría una eternidad, en términos atómicos, para aproximarse a los tamaños que describen Kroto y Smalley, dado su limitado número de enlaces.

Otra cosa aún más importante: Ebert señala que el hollín real que se forma en una llama real contiene muchos otros elementos, además del carbono, que entorpecerían la formación del futbolano como: el oxígeno, hidrógeno, nitrógeno o azufre. Estos cubririan los enlaces libres, impidiendo que se unieran otros carbonos $y$, por tanto, se formara la curvatura en las láminas. Ebert piensa que ha conseguido producir una nueva prueba de la existencia del modelo tradicional del hollín con técnicas de difracción por rayos $X$, en las cuales, éstos pasan a través de las moléculas y se analizan sus patrones de dispersión. Las lecturas de los rayos $\mathrm{X}$ indican que la mayor parte del hollín está hecho de estratos, que contienen un promedio de 133 átomos de carbono, rodeados por oxígeno, hidrógeno, carbono y azufre, los cuales se apilan para formar moléculas más grandes.

Esto podría significar el golpe de gracia para los teóricos del futbolano. Una cosa es deducir la forma de una molécula, basándose en la expulsión de unos pocos átomos de $\mathrm{C}_{2}$, y otra conseguir un retrato en rayos $\mathrm{X}$ de esta sustancia. Sin embargo, Smalley no piensa que los trabajos de Ebert contradigan los suyos. Según él, es muy posible que las partículas de hollín incluyan otros átomos yadopten otras formas, pero sólo en sus últimos estados de formación. "En cuanto la espiral toma cierto tamaño", dice Smalley, "la posibilidad de tener un defecto en su estructura, como sería un hidrógeno o una brecha, aumenta hasta el punto de que puede hacer desaparecer la espiral. No me sorprende que hayan grandes cantidades de oxígeno e hidrógeno en las últimas vueltas de estas estructuras. Pero de lo que yo estoy hablando es de las primeras vueltas de la formación, y ahí sus mediciones no pueden llegar".

El químico alemán Klaus Homann, del Instituto de Química y Física de Darmstadt, defiende al futbolano, pero está a caballo entre las dos posturas. Dice que realmente existe algo parecido al buckminterfullereno, pero que no se forma tal y como proponen Smalley y Kroto. Homann estudió una llama de gas, y descubrió que la zona de oxidación estaba, en efecto, llena de $\mathrm{C}_{60^{\circ}}$ Pero opina que estas moléculas son, en realidad, balones poliédricos. El hollín crecería en un apilamiento desordenado de láminas de carbono. En un momento determinado se forma una burbuja en la lámina superior, que consta de un sólo pentágono de carbono rodeado por cinco hexágonos de carbono. En el extremo abierto de la burbuja se unen más átomos, para alcanzar una forma termodinámicamente más estable. Finalmente, los carbonos cierran la esfera, permitiendo que ésta se libere de la lámina y quede a la deriva.

\section{CONCLUSION}

El trabajo de Homann ha sido una noticia bien recibida por ambos bandos. Aunque ninguna de las dos teorías ha quedado justificada, todos han sacado algo de esta investigación. Eberty Frenklach la interpretan como un voto a favor de estos futbolanos de formación tardia, como un mero producto del hollín. Smalley cree que, tanto esto como las diferencias con Homann, es una cuestión de interpretación. La única conclusión a la que han llegado es que los mecanismos de formación son diferentes. Según Smalley, el que propone Homann es más complicado y menos plausible.

Las implicaciones del futbolano van más allá de la formación del hollín: las condiciones de la corteza exterior de las estrellas rojas gigantes, ricas en carbono, son prácticamente idénticas a la zona de formación del hollín de una vela: la misma temperatura y combinación de elementos. Si los futbolanos realmente se producen en las llamas de las velas, también podrian producirse en las estrellas rojas gigantes. Durante millones de años, las moléculas suspendidas en las proximidades de las estrellas habrian sido bombardeadas por radiaciones solares. Las moléculas libres de mayor tamaño, posiblemente no resistieron y fueron destruidas. Perolos futbolanos habrian sobrevivido yestarian pululando por el cosmos. 
Es evidente que nadie puede enviar un espectrómetro de masa al espacio para observar a los buckminsterfullerenos, así que hay que utilizar otros medios. Kroto y Smalley creen poder probarlo con la forma de las zonas interestelares difusas -ZID- . Desde los años 30 , los astrónomos han notado la existencia de aspectos extraños en los espectros de luz de las estrellas lejanas; algo está absorbiendo ciertas frecuencias de luz, cuando pasan de las estrellas a la tierra. Sin embargo, ese algo no se corresponde con las líneas del espectro de ninguna molécula conocida. Según Kroto: "Sea lo que sea, se trata de un material que satura el espacio que hay entre las estrellas, el cual no hemos sido nunca capaces de hallar. Lo que es seguro es que abunda por todas partes".

Kroto y Smalley sospechan que se trata de Bucky. Pero también son conscientes de lo difícil que será demostrarlo. Primero tendrían que producirfutbolanos suficientes como para determinar una señal del espectro característico, y poder entonces comparar estas líneas con las de las ZID. Sólo consiguiendo esta especie de huella dactilar celeste podrían demostrar la omnipresencia del futbolano. Frenklachestá convencido de lo contrario. En un reciente experimento, que este último ha hecho, analizó metano, argón, hidrógeno y silano - materiales presentes en las estrellas ricas en carbono - a más de 4900 grados. Tal y como espiraba, se produjo una especie de hollín celeste. Al menos en el laboratorio el humo de las estrellas no parece ser de futbolanos, sino de diminutas partículas de carburo de silicio.

Smalley no considera que estas conclusiones sean convincentes: "No se puede deducir de ese experimento que el carburo de silicio se forme a esa temperatura límite. Todo lo que se sabe es que, cuando los materiales se enfriaron, esa sustancia estaba alli. Eso no contrasta con que el carburo de silicio pueda formarse a altas temperaturas estelares".

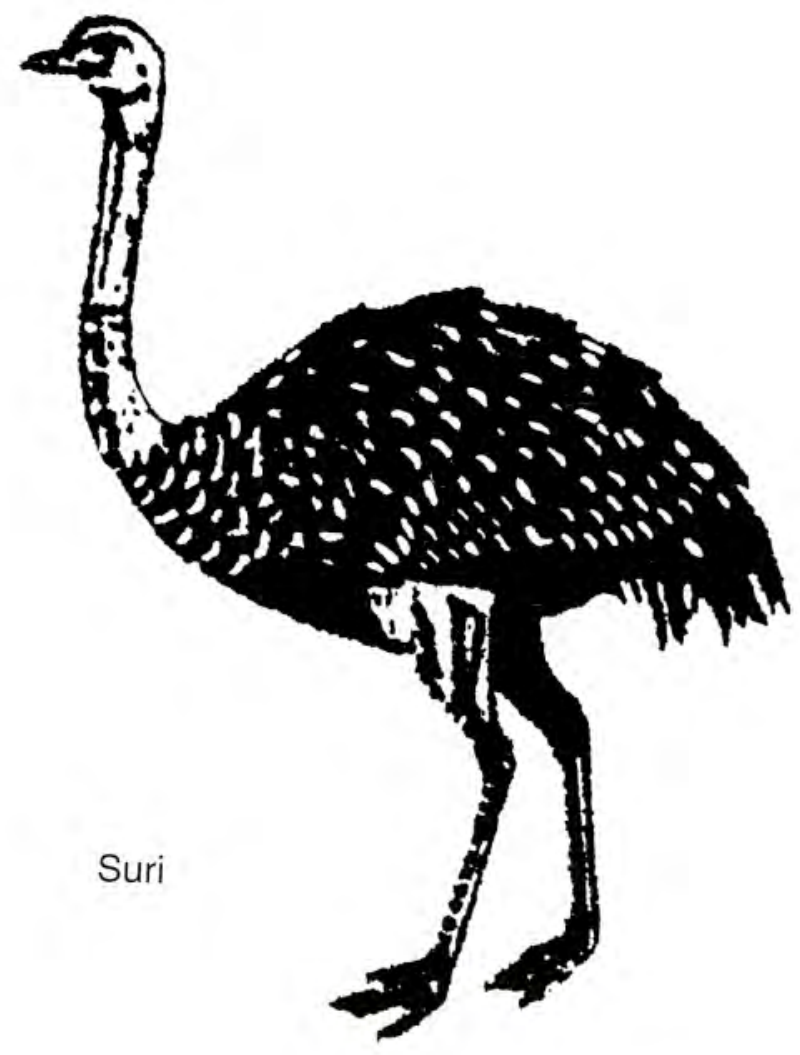

